\title{
Deep-blue organic light-emitting diodes based on a doublet $d-f$ transition cerium(III) complex with $100 \%$ exciton utilization efficiency
}

\author{
Liding Wang ${ }^{1}$, Zifeng Zhao (1)', Ge Zhan ${ }^{1}$, Huayi Fang ${ }^{2}$, Hannan Yang ${ }^{3}$, Tianyu Huang ${ }^{4}$, Yuewei Zhang ${ }^{4}$, Nan Jiang ${ }^{3}$,
} Lian Duan", Zhiwei Liu (1)', Zuqiang Bian', Zhenghong Lu $\mathbb{B}^{3}$ and Chunhui Huang ${ }^{1}$

\begin{abstract}
Compared to red and green organic light-emitting diodes (OLEDs), blue OLEDs are still the bottleneck due to the lack of efficient emitters with simultaneous high exciton utilization efficiency (EUE) and short excited-state lifetime. Different from the fluorescence, phosphorescence, thermally activated delayed fluorescence (TADF), and organic radical materials traditionally used in OLEDs, we demonstrate herein a new type of emitter, cerium(III) complex Ce-1 with spin-allowed and parity-allowed $d-f$ transition of the centre $\mathrm{Ce}^{3+}$ ion. The compound exhibits a high EUE up to $100 \%$ in OLEDs and a short excited-state lifetime of $42 \mathrm{~ns}$, which is considerably faster than that achieved in efficient phosphorescence and TADF emitters. The optimized OLEDs show an average maximum external quantum efficiency (EQE) of $12.4 \%$ and Commission Internationale de L'Eclairage (CIE) coordinates of $(0.146,0.078)$.
\end{abstract}

\section{Introduction}

Organic light-emitting diodes (OLEDs) have been successfully commercialized in the niche display market and are now under intense research for other applications, such as solid-state lighting. During the past three decades, fluorescence $^{1}$, phosphorescence ${ }^{2-5}$, thermally activated delayed fluorescence (TADF $)^{6-8}$, and organic radical ${ }^{9-11}$ materials have been subsequently applied as emitters because of their high efficiency, long-term stability, and low cost. As a new type of emitter in OLEDs, cerium(III) complexes have many potential advantages. First, we propose that the theoretical exciton utilization efficiency (EUE) could be as high as $100 \%$, since the cerium(III) complex shows a doublet $5 d-4 f$ transition of the single

\footnotetext{
Correspondence: Zhiwei Liu (zwliu@pku.edu.cn)

${ }^{1}$ Beijing National Laboratory for Molecular Sciences (BNLMS), State Key Laboratory of Rare Earth Materials Chemistry and Applications, Beijing Engineering Technology Research Centre of Active Display, College of Chemistry and Molecular Engineering, Peking University, 100871 Beijing, China ${ }^{2}$ Tianjin Key Lab for Rare Earth Materials and Applications, School of Materials Science and Engineering, Nankai University, 300350 Tianjin, China Full list of author information is available at the end of the article These authors contributed equally: Liding Wang, Zifeng Zhao
}

electron of the centre $\mathrm{Ce}^{3+}\left(4 f^{\mathrm{d}}\right.$ configuration) ion rather than a singlet and/or triplet transition, which will not be limited by spin statistics ${ }^{10,11}$. Second, cerium(III) complexes are expected to be more stable in OLEDs since their excited-state lifetimes are generally tens of nanoseconds $^{12-15}$. Third, cerium(III) complexes are inherent blue or ultraviolet emitters, as demonstrated in the literature, although their emission colours could be theoretically affected by the ligand field ${ }^{16}$. Moreover, cerium (III) complexes are inexpensive because the abundance of cerium in Earth's crust is $0.006 \mathrm{wt} \%$, which is four orders of magnitude higher than that of iridium $(0.0000001 \mathrm{wt} \%)$ and even slightly higher than that of copper $(0.005 \mathrm{wt} \%)^{17}$.

However, most reported cerium(III) complexes are nonemissive because classic ligands and solvent molecules are found to quench $\mathrm{Ce}^{3+}$ ion luminescence upon coordina$\operatorname{tion}^{18}$. Hence, electroluminescence (EL) studies on cerium(III) complexes are very rare, and their advantages have not been demonstrated. To date, there are only three examples of EL study of cerium(III) complexes in the literature $^{19-21}$. Among these examples, the maximum external quantum efficiency (EQE) of the best result is

\section{(c) The Author(s) 2020}

(c) (i) Open Access This article is licensed under a Creative Commons Attribution 4.0 International License, which permits use, sharing, adaptation, distribution and reproduction cc) in any medium or format, as long as you give appropriate credit to the original author(s) and the source, provide a link to the Creative Commons license, and indicate if changes were made. The images or other third party material in this article are included in the article's Creative Commons license, unless indicated otherwise in a credit line to the material. If material is not included in the article's Creative Commons license and your intended use is not permitted by statutory regulation or exceeds the permitted use, you will need to obtain permission directly from the copyright holder. To view a copy of this license, visit http://creativecommons.org/licenses/by/4.0/. 
below 1\%. As a breakthrough, we report herein a novel and neutral cerium(III) complex Ce-1 with rigid scorpionate ligands showing a high photoluminescence quantum yield (PLQY) up to $93 \%$ in doped film and consequently a high average EQE of $12.4 \%$ in prototype OLEDs.

\section{Results}

\section{Synthesis and structure}

The complex Ce-1 was synthesized by stirring potassium hydrotris(3,5-dimethylpyrazolyl)borate $\left(\mathrm{KTp}^{\mathrm{Me} 2}\right)^{22}$ with $\mathrm{Ce}\left(\mathrm{CF}_{3} \mathrm{SO}_{3}\right)_{3}$ in tetrahydrofuran (THF), accompanied by hydrolysis due to a trace amount of water in the solvent (Fig. 1a). Though the reaction was found accidentally, it is repeatable and stable by adopting the conditions presented in the experimental section. Intriguingly, when $\mathrm{KTp}^{\mathrm{Me} 2}$ was replaced by potassium trispyrazolylborate (KTp) in this reaction, the expected complex $\mathrm{Ce}(\mathrm{Tp})_{3}$ was successfully obtained. The molecular structure and photoluminescence (PL) spectrum are exhibited in Supplementary Fig. S1. These results indicate that the methyl group on the pyrazole group has a crucial influence on the reaction. Most likely, the huge steric hindrance of the methyl group makes $\mathrm{Ce}\left(\mathrm{Tp}^{\mathrm{Me} 2}\right)_{3}$ unstable and tend to react with trace amounts of water in the solvent to form hydrolysate $\mathbf{C e - 1}$.

The thermal stability of $\mathbf{C e - 1}$ is favourable, showing a decomposition temperature $\left(T_{\mathrm{d}}\right.$, corresponding to a $1 \%$ weight loss) of $265^{\circ} \mathrm{C}$ (Supplementary Fig. S2). Hence, the complex Ce-1 was purified by thermal gradient sublimation at $230^{\circ} \mathrm{C}$ and $2 \times 10^{-4} \mathrm{~Pa}$, during which single crystals were collected. The sublimated $\mathbf{C e}-\mathbf{1}$ was identified by high-resolution mass spectrometry, elemental analysis, and single crystal X-ray diffraction. No ${ }^{1} \mathrm{H}$ NMR data were collected since Ce-1 is paramagnetic, as demonstrated by electron paramagnetic resonance (EPR) spectroscopy (Supplementary Fig. S3). There are two almost identical coordination environments for $\mathrm{Ce}^{3+}$ ions in the crystal. The central $\mathrm{Ce}^{3+}$ ions are coordinated by tridentate and pentadentate ligands with one and two negative charges, respectively. There are seven nitrogen atoms and one oxygen atom surrounding cerium in Ce-1, with $\mathrm{Ce}-\mathrm{N}$ distance in the range of $2.573-2.680 \AA$ and a Ce-O distance of $\sim 2.399 \AA$ (Supplementary Table S1). Figure 1b, c shows one of the structures in ellipsoid and space-fill styles. From the space-fill model, it is found that
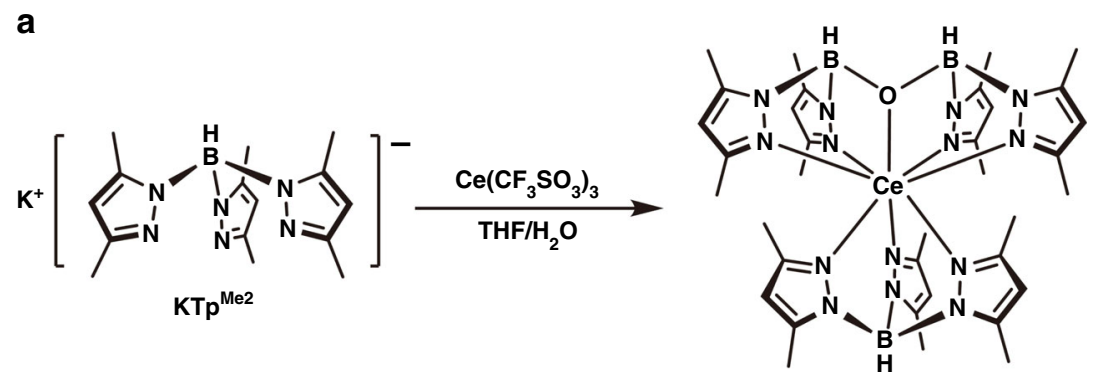

Ce-1
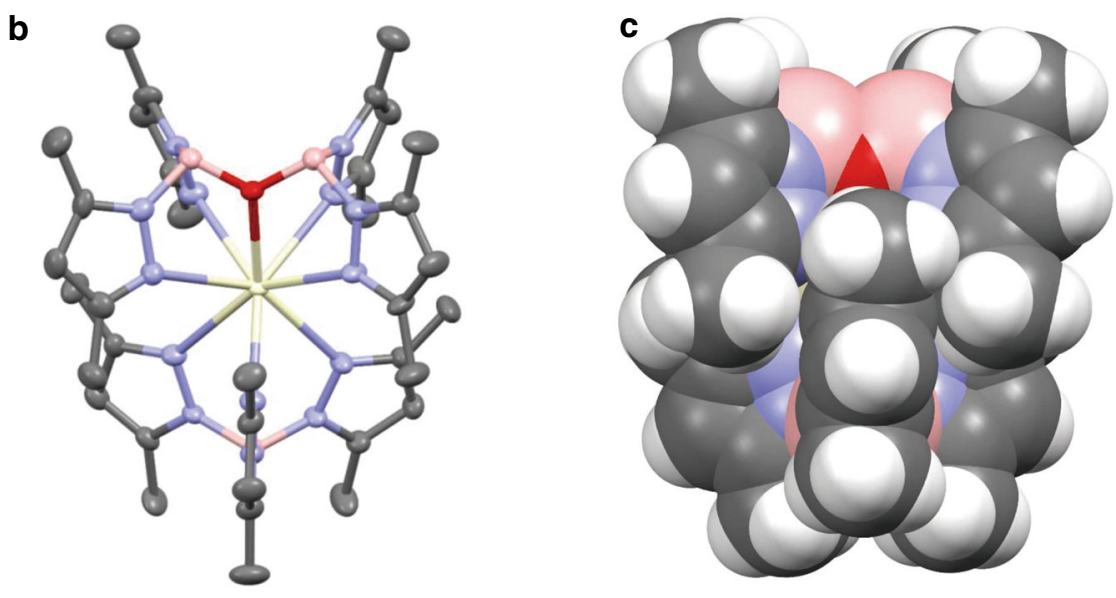

Fig. 1 Synthesis and structure of the investigated cerium(III) complex Ce-1. a Synthetic route for the complex. b Single crystal structure of the complex shown as ellipsoids at the $50 \%$ probability level, where yellow represents Ce, pink represents B, blue represents N, red represents $\mathrm{O}$, grey represents $C$, and the hydrogens are omitted for clarity. c Single crystal structure of the complex shown in a space-fill style, where hydrogens are shown in white 
the ligands shield the $\mathrm{Ce}^{3+}$ ion completely, which is important for high PL efficiency, since coordinating solvent molecules are frequently efficient quenchers of the lanthanide luminescence ${ }^{23}$.

\section{Photophysical properties}

Figure 2a shows UV-Vis absorption spectra of Ce-1 and $\mathrm{KTp}^{\mathrm{Me} 2}$ in DCM solution. The three bands at 373,322 , and $280 \mathrm{~nm}$ with a molar extinction coefficient $(\varepsilon)$ of $\sim 10^{2}$ $\mathrm{L} \mathrm{mol}^{-1} \mathrm{~cm}^{-1}$ can be assigned to the $4 f-5 d$ transitions of the $\mathrm{Ce}^{3+}$ ion by comparing the absorption spectra of $\mathbf{C e - 1}$ and $\mathrm{KTp}^{\mathrm{Me} 2}$. Another absorption band below $260 \mathrm{~nm}$ originates from the $\pi-\pi^{*}$ transition of the ligand. The excitation spectrum of Ce-1 in DCM (Supplementary Fig. S4) has a similar pattern to its absorption spectrum. The peak at $\sim 240 \mathrm{~nm}$ is attributed to ligand absorption, exhibiting cascade-type energy transfer from the ligand to the central $\mathrm{Ce}^{3+}$ ion under high-energy excitation. Due to parity-allowed characteristics, the complex can also be easily excited by direct $f-d$ absorption (the peak at $\sim 375 \mathrm{~nm}$ ), while $f-f$ excitation is difficult in a dilute solution of a typical $\mathrm{Eu}$ (III) complex due to the parityforbidden characteristics of $f-f$ absorption.

The dilute DCM solution and crystalline powder of Ce-1 both exhibit strong blue emission. Their PL spectra (Fig. 2a) show two broad bands with different relative amplitudes. The redshift can be ascribed to molecule packing in crystals, while the relative amplitude may be sensitive to the coordination environment ${ }^{24}$. At $77 \mathrm{~K}$, the emission spectrum of the crystalline powder splits into two peaks at 436 and $477 \mathrm{~nm}$. The energy difference between the two peaks is close to $2000 \mathrm{~cm}^{-1}$, in agreement with the energy splitting between ${ }^{2} \mathrm{~F}_{5 / 2}$ and ${ }^{2} \mathrm{~F}_{7 / 2}$, two ground levels of the $\mathrm{Ce}^{3+}$ ion $^{20}$. Moreover, the decay lifetimes of the solution at room temperature, crystalline powder at room temperature and crystalline powder at $77 \mathrm{~K}$ were measured to be approximately 53,42 , and $42 \mathrm{~ns}$ (Fig. 2b), respectively, which are comparable with those of previously reported cerium(III) complexes ${ }^{12-14}$. In addition, the emissions of $\mathbf{C e - 1}$ in solvents with different polarities showed almost identical spectra (Supplementary Fig. S5). This illustrates that unlike the famous radical emitter TTM-3NCz ${ }^{11}$, the electron transition in $\mathbf{C e - 1}$ is highly localized.

The PLQYs of Ce-1 in dichloromethane (DCM, $10^{-5}$ $\mathrm{M})$ and as crystalline powder are $48 \%$ and $82 \%$, respectively. It should be noted that the PLQYs of Ce-1 in DCM can be promoted by increasing the concentration, reaching $\sim 100 \%$ at $10^{-3} \mathrm{M}$ (Supplementary Table S2). By combining the lifetime and PLQY values, the radiative and nonradiative decay rates $\left(k_{\mathrm{r}}\right.$ and $\left.k_{\mathrm{nr}}\right)$ are calculated as $9.1 \times 10^{6}$ and $9.8 \times 10^{6} \mathrm{~s}^{-1}$ for the DCM solution at $10^{-5}$ $\mathrm{M}$ and as $2.0 \times 10^{7}$ and $4.3 \times 10^{6} \mathrm{~s}^{-1}$ for the crystalline powder, respectively. The higher PLQY in the solid state is attributed to both an increased radiative rate constant and a decreased nonradiative rate constant, indicating that rigid molecular packing is beneficial for promoting radiative transition and inhibiting nonradiative transition. In addition, these values are comparable to those of organic radical materials and an order of magnitude larger than those of phosphorescence and TADF materials.

To obtain more in-depth insights into the excited state of Ce-1, theoretical calculations were carried out (Supplementary Fig. S6). The donor and acceptor for the first symmetry allowed transition were recognized as the $4 f$ and $5 d_{z}^{2}$ orbitals, respectively. This result further proves
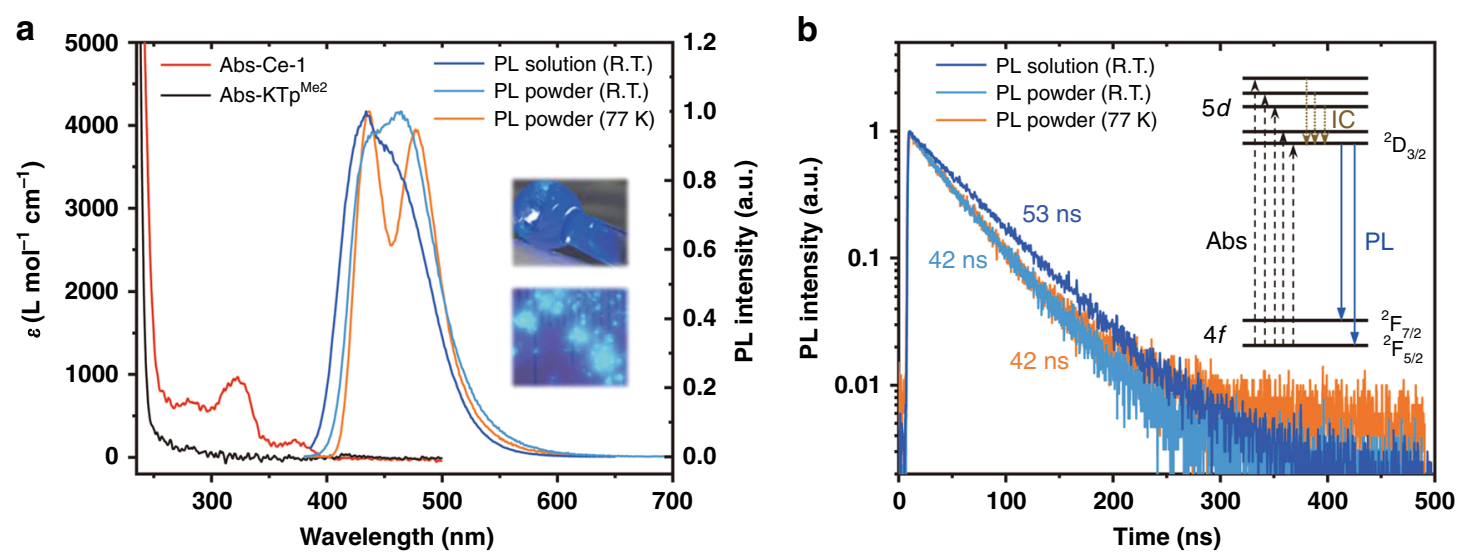

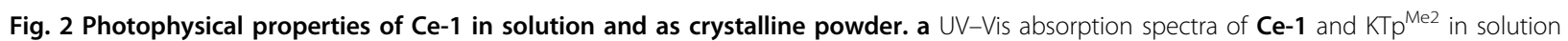
$\left(\mathrm{DCM}, 10^{-5} \mathrm{M}\right)$, and PL spectra of Ce-1 in solution (DCM, $\left.10^{-5} \mathrm{M}\right)$ and as crystalline powder at room temperature (RT) and $77 \mathrm{~K}$. The excitation wavelength is $370 \mathrm{~nm}$. Inset, photographs of the complex in solution and as crystalline powder under $365 \mathrm{~nm}$ irradiation. $\mathbf{b}$ Transient PL decays of the complex in solution and as crystalline powder at room temperature and $77 \mathrm{~K}$. The excitation wavelength is $375 \mathrm{~nm}$. Inset, schematic diagram to illustrate the PL mechanism of the cerium(III) complex, where Abs, IC, and PL represent absorption, internal conversion, and photoluminescence, respectively. ${ }^{2} \mathrm{D}_{3 / 2}$ is the lowest excited state of the $\mathrm{Ce}^{3+}$ ion. ${ }^{2} \mathrm{~F}_{5 / 2}$ and ${ }^{2} \mathrm{~F}_{7 / 2}$ are two ground levels of the $\mathrm{Ce}^{3+}$ ion 
the transition mechanism of Ce-1. Therefore, the observed strong blue emission can be attributed to the $\mathrm{Ce}^{3+}$ ion, more specifically to the two electric-dipole $5 d-4 f$ transitions of the $\mathrm{Ce}^{3+}$ ion from the lowest excited state $\left({ }^{2} \mathrm{D}_{3 / 2}\right)$ to the ground state ${ }^{2} \mathrm{~F}_{5 / 2}$ and ${ }^{2} \mathrm{~F}_{7 / 2}$, as shown in the inset of Fig. 2b.

\section{EL properties}

The Ce-1 complex shows high PLQY, excellent thermal stability, good stability in air (Supplementary Fig. S7), and short excited-state lifetime; it is worth investigating as the emitter in OLEDs with a vacuum thermal deposition method. The frontier molecular orbital energy levels of Ce-1 were obtained as $-6.2 \mathrm{eV}$ for the highest occupied molecular orbital (HOMO) and $-3.1 \mathrm{eV}$ for the lowest unoccupied molecular orbital (LUMO) by investigating the ultraviolet photoelectron spectrum (Supplementary Fig. S8) and UV-Vis absorption spectrum. To optimize the OLED structure, the theoretical equation for calculating the EQE of OLEDs should be considered

$$
\mathrm{EQE}=\mathrm{n}_{\mathrm{e}-\mathrm{h}} \times \mathrm{n}_{\mathrm{PL}} \times \mathrm{n}_{\text {out }}
$$

where $\eta_{e-h}$ is the recombination efficiency of injected holes and electrons, $\mathrm{n}_{\mathrm{PL}}$ is the intrinsic PL efficiency, i.e., the PLQY of the emission layer, $\eta_{\text {exciton }}$ is the EUE, i.e., the ratio of radiative excitons to total formed excitons, and $\eta_{\text {out }}$ is the light out-coupling efficiency. Based on the equation, the key is to screen host and carrier transport materials, hence achieving high PLQY in thin Ce-1 film, and to balance and confine hole and electron recombination within/near the emission layer.

First, bis[4-(N-carbazolyl)-phenyl]phenylphosphine oxide (BCPO) was chosen as the host due to its high triplet energy level and excellent bipolar carrier mobility. Device D1 was fabricated with a structure of indium tin oxide (ITO) $/ \mathrm{MoO}_{3}(2 \mathrm{~nm}) / \mathrm{MoO}_{3}$-doped 9-(4-tert-butylphenyl)-3,6-bis(triphenylsilyl)-9H-carbazole ( $\mathrm{CzSi:MoO}$, $20 \mathrm{wt} \%, \quad 10 \mathrm{~nm}) / \mathrm{CzSi} \quad(30 \mathrm{~nm}) / \mathrm{BCPO}: \mathrm{Ce}-1 \quad(10 \mathrm{wt} \%$, $20 \mathrm{~nm}$ )/diphenyl-4-triphenylsilylphenyl-phosphine oxide (TSPO1, $10 \mathrm{~nm}) / 2,2^{\prime}, 2^{\prime \prime}$-(1,3,5-benzinetriyl)-tris(1-phenyl-1- $H$-benzimidazole) $\quad(\mathrm{TPBi}, 40 \mathrm{~nm}) / \mathrm{LiF} \quad(0.7 \mathrm{~nm}) / \mathrm{Al}$ $(100 \mathrm{~nm})$. The frontier orbital energy levels of the utilized materials ${ }^{25-27}$ are shown in Fig. 3a. The device characteristics are plotted in Fig. 3b, c. The device emits deepblue light similar to the Ce-1 solution with CIE coordinates of approximately $(0.15,0.08)$ (Supplementary Fig. S9). Considering that the doped BCPO:Ce-1 (10 wt\%) film has a moderate PLQY of $26 \%$, the device shows a relatively good maximum EQE of 5.1\% and low efficiency rolloff (Fig. 3b). To estimate the EUE of D1 more accurately, we used the variable-angle spectroscopic ellipsometry (VASE) method to determine the molecular orientation distribution in the emission layer (Supplementary Fig. S10 and Table S3) ${ }^{28}$. The Ce-1 molecules show a horizontal dipole ratio of $68.1 \%$ in the BCPO film, which is close to the randomly oriented ratio $(66.7 \%)^{29,30}$, indicating $\sim 20 \%$ $\mathrm{y}_{\text {out }}$, and hence, an $100 \%$ EUE is demonstrated in D1. Thus, we expect that the performance of our deep-blue OLEDs could be further improved by screening the host material to obtain a higher PLQY.

Therefore, a series of Ce-1-doped films were fabricated in a vacuum chamber and subsequently measured for PLQYs and PL spectra (Supplementary Fig. S11 and Fig. 3e). Among these films, the TSPO1:Ce-1 (10 wt\%), CzSi:Ce-1 (10 wt\%), and TSPO1:CzSi:Ce-1 (0.18:0.72:0.1 in weight ratio) films show relatively high PLQYs up to $70 \%, 83 \%$, and $93 \%$, respectively. With substantial device optimization (see Supplementary Table S4 for details), a champion performance was obtained for D2 with the structure of $\mathrm{ITO} / \mathrm{MoO}_{3}(2 \mathrm{~nm}) / \mathrm{CzSi}: \mathrm{MoO}_{3}(20 \mathrm{wt} \%$, $30 \mathrm{~nm}) / \mathrm{CzSi}(10 \mathrm{~nm}) / \mathrm{TSPO} 1 \mathrm{CzSi}$ Ce-1 (0.18:0.72:0.1 in weight ratio, $20 \mathrm{~nm}) /$ TSPO1 $(10 \mathrm{~nm}) /$ bathophenanthroline (Bphen, $40 \mathrm{~nm}) / \mathrm{LiF} \quad(0.7 \mathrm{~nm}) / \mathrm{Al} \quad(100 \mathrm{~nm})$. The device characteristics are plotted in Fig. 3b, c for comparison. The champion performance includes a turn-on voltage of $3.6 \mathrm{~V}$, a high maximum EQE of $14.0 \%$, and a typical emission from Ce-1 with CIE coordinates of $(0.146,0.078)$. This result is comparable to the best reported deep-blue OLEDs with a platinum complex, an iridium complex or TADF as the emitter (Supplementary Table S5) ${ }^{31-33}$. To estimate the EUE of D2, we measured the horizontal dipole ratio of $\mathbf{C e - 1}$ in TSPO1: $\mathrm{CzSi}$ (0.18:0.72 in weight ratio) as only 56.0\% (Supplementary Fig. S10 and Table S3), which is even lower than that of the random orientation (66.7\%), leading to an $\mathrm{n}_{\text {out }}$ lower than $20 \%$. Considering the PLQY of $93 \%$ and EQE of $14.0 \%$, the EUE of D2 is deduced to be higher than $70 \%$ (Table 1).

The details of carrier recombination are critical for explaining the high EUE (100\% for D1 and $>70 \%$ for D2) of these devices. As depicted in Fig. 3e, Supplementary Figs. S9 and S11, the steady-state PL spectra of emission layers contain deep-blue emissions that are substantially identical to the solution emission of $\mathbf{C e - 1}$ and weak ultraviolet emissions from host materials, indicating inefficient energy transfer from hosts to Ce-1. In contrast, the EL spectra of these devices exhibit pure emissions from Ce-1, implying that carrier recombination dominantly occurs on Ce-1 rather than on host molecules. This inference can be further confirmed by the transient EL spectrum of D2 (Fig. 3f). The spike (in the red dashed square) is proven to be evidence of charge trapping on the guest $^{34}$. Interestingly, the response time of the EL intensity to the applied electric field is slow, implying that there may be a slow process of charge migration and carrier trapping in D2. After the polarity of the electric field is reversed, the remaining carriers in the device de-trap and 

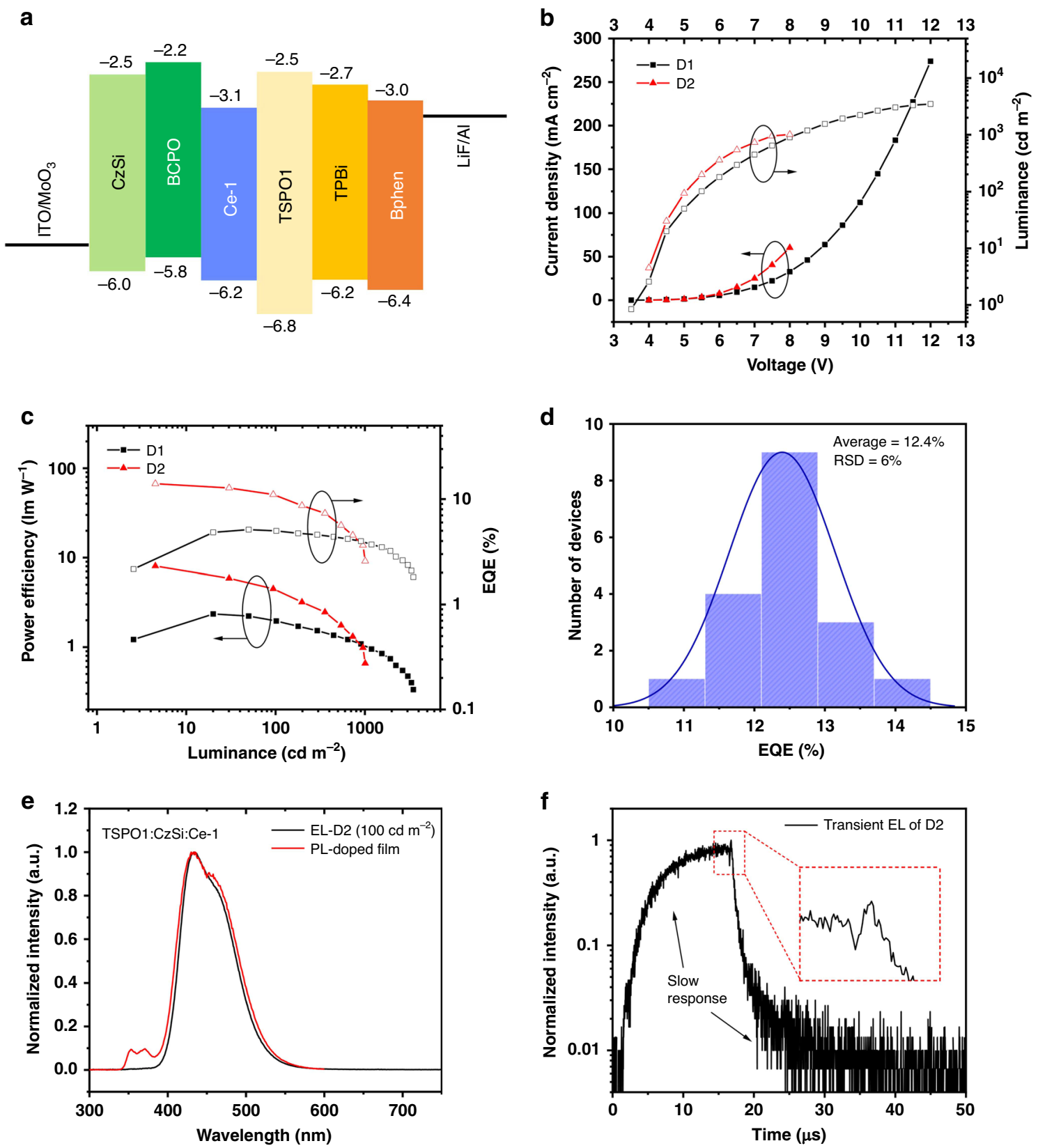

Fig. 3 OLED demonstration. a Frontier orbital energy levels for materials used in OLEDs; the energy levels of Ce-1 were deduced from its ultraviolet photoelectron spectrum and ultraviolet absorption spectrum. b Current density-voltage-luminance traces for the key OLEDs. c Power efficiency-luminance-EQE traces for the key OLEDs. d Maximum EQE histogram of 18 devices with the optimized device structure (average, 12.4\%; highest, 14.0\%). e PL spectrum of the TSPO1:CzSi:Ce-1 (0.18:0.72:0.1 in weight ratio, excitation $280 \mathrm{~nm})$ film, and EL spectrum of D2 at the luminance of $100 \mathrm{~cd} \mathrm{~m}^{-2}$. $\mathbf{f}$ Transient EL spectrum of D2

continue to recombine on the guest, resulting in a microsecond delay that is much longer than the excitedstate lifetime of Ce-1. Moreover, the absorption edge of the ligand is $\sim 250 \mathrm{~nm}$, corresponding to a bandgap of $\sim 5 \mathrm{eV}$. However, the turn-on voltages of D1 and D2 are in the range of 3.6-3.8 $\mathrm{V}$, which are much lower than the bandgap of the ligand. This implies that carriers recombine on the $\mathrm{Ce}^{3+}$ ions with a narrower bandgap instead of on the ligands. The speculated EL mechanism is exhibited in Fig. 4. Thus, doublet exciton formation is dominant in these OLEDs. Since Ce(III) ions are more likely to lose only one electron from the $4 f$ orbital to form $\mathrm{Ce}(\mathrm{IV})$, the bottom route would be the main route for exciton formation in the device. 
Table 1 Summarized parameters of key OLEDs with Ce-1 as the emitter

\begin{tabular}{|c|c|c|c|c|c|c|}
\hline Device & Host & $V_{\text {on }}{ }^{\mathrm{a}}[\mathrm{V}]$ & $\mathrm{EQE}_{\max }{ }^{\mathrm{b}}[\%]$ & $\mathrm{CE}_{\max }{ }^{\mathrm{c}}\left[\mathrm{cd} \mathrm{A}{ }^{-1}\right]$ & $L_{\max }{ }^{d}\left[c d m^{-2}\right]$ & $\mathrm{CIE}^{\mathrm{e}}$ \\
\hline \multirow[t]{3}{*}{ D1 } & $\mathrm{BCPO}$ & & & & & $(0.148,0.083)$ \\
\hline & Average $^{f}$ & 3.6 & 5.0 & 3.4 & 3457 & \\
\hline & Champion & 3.6 & 5.1 & 3.5 & 3494 & \\
\hline \multirow[t]{3}{*}{ D2 } & TSPO1:CzSi & & & & & $(0.146,0.078)$ \\
\hline & Average $^{g}$ & 3.8 & 12.4 & 8.7 & 948 & \\
\hline & Champion & 3.6 & 14.0 & 10.3 & 1008 & \\
\hline
\end{tabular}

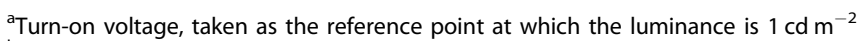

${ }^{\mathrm{b}}$ Maximum EQE

${ }^{\mathrm{C}}$ Maximum current efficiency

${ }^{\mathrm{d}}$ Maximum luminance

${ }^{\text {e}}$ Coordinates at $100 \mathrm{~cd} \mathrm{~m}^{-2}$

${ }^{\mathrm{f}}$ Average of three devices

${ }^{\mathrm{g}}$ Average of 18 devices

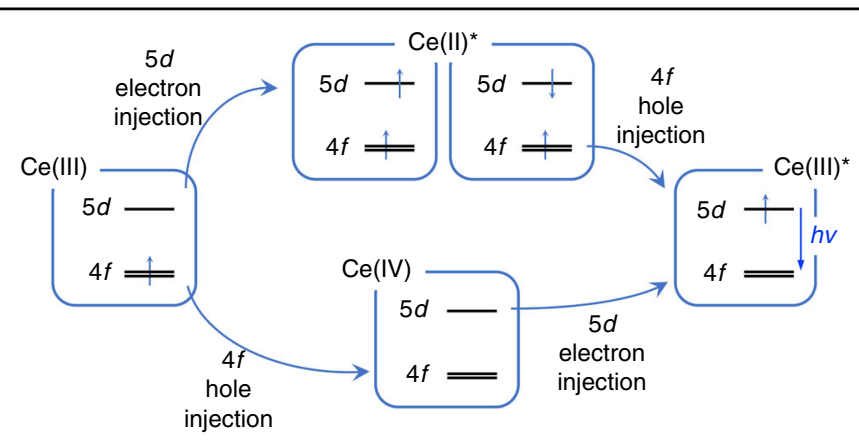

Fig. 4 Speculated electroluminescence mechanism. The electron or hole is captured by a Ce(III) ion to form Ce(II)* (top route) or Ce(IV) (bottom route); then, hole or electron injection brings the intermediate species to the excited $\mathrm{Ce}(I I I)^{*}$ ion state

Although device D2 shows high efficiency, the maximum luminance of D2 is only $1008 \mathrm{~cd} \mathrm{~m}^{-2}$, and the efficiency roll-off is severe, which is contrary to the common knowledge that a short excited-state lifetime emitter is beneficial for reducing efficiency roll-off. In addition, the lifetime $\left(\mathrm{LT}_{50}\right)$ of $\mathbf{D} 2$ was measured as only 147 s (Supplementary Fig. S12). To obtain more in-depth insights into the device efficiency roll-off and stability, we measured device D2 over three cycles and found that the device performance rapidly degrades (Fig. 5), indicating that the efficiency roll-off should be ascribed to the instability of the device. Furthermore, we introduced a florescence deep-blue emitter, 1-(10-(4-methoxyphenyl) anthracen-9-yl)-4-(10-(4-cyanophenyl)anthracen-9-yl)tetraphenylethene (TPEA) ${ }^{35}$, and a classic host material, 1,3bis(N-carbazolyl)benzene, as references to fabricate control devices (Supplementary Fig. S12). Detailed results indicate that the instability of the host material, especially TSPO $1^{36-38}$, is an important reason for device degradation. In addition, the $\mathrm{Ce}$ (III) complex is known to be a strong photo-reductant. This could be another reason for the device instability. Thus, the stability of Ce-1 in terms of EL remains to be explored.

\section{Discussion}

Materials with doublet emission from single-electron transition could endow OLEDs with $\sim 100 \%$ EUE and a short excited-state lifetime (tens of nanoseconds, close to that of fluorescence materials). Organic radical emitters have achieved highly efficient doublet deep-red devices ${ }^{11}$. In this work, we have demonstrated a high EQE in deepblue OLEDs based on a new cerium(III) complex Ce-1 as the emitter, which shows parity-allowed doublet $d-f$ transition of the centre $\mathrm{Ce}^{3+}$ ion with similar advantages in OLEDs. Interestingly, the organic radical emitter is beneficial for obtaining OLEDs with low-energy transitions, such as red and infra-red ${ }^{39}$, while the $d-f$ transitionbased $\mathrm{Ce}$ (III) complex is good for achieving OLEDs with high-energy transitions, such as blue and ultraviolet. Although the $147 \mathrm{~s}$ device lifetime is short, we believe this is a good start for deep-blue $(\mathrm{CIEy}<0.1)$ devices, especially considering that no device lifetime of such deep- 

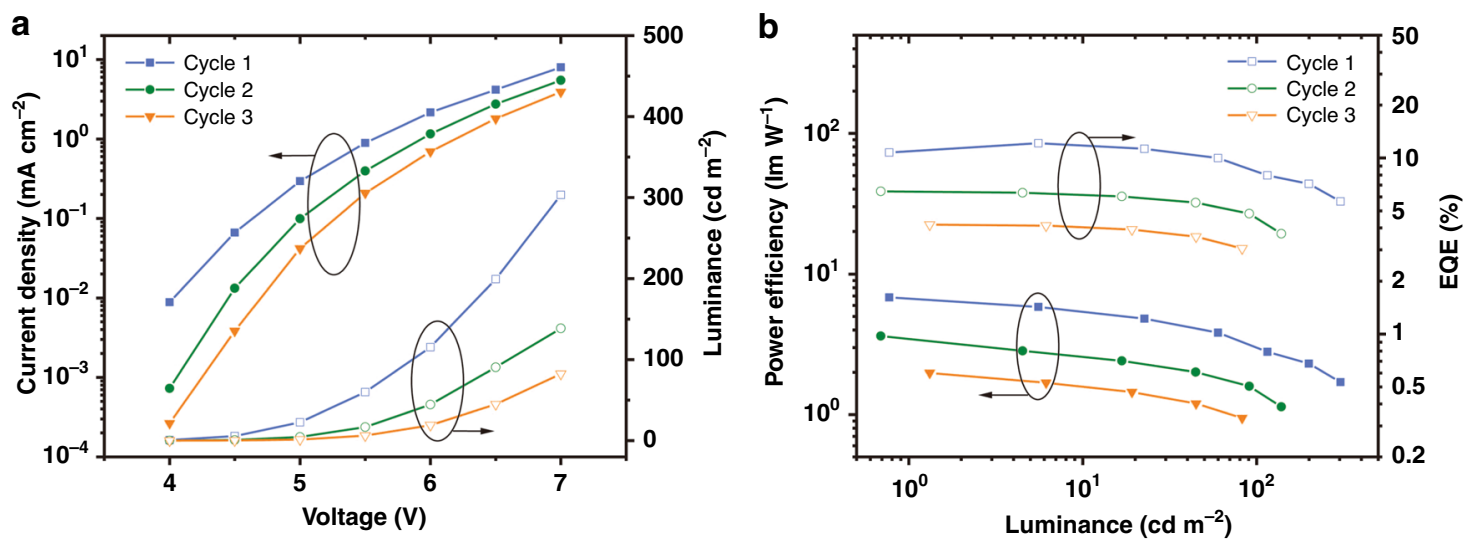

Fig. 5 Cycling demonstration of device D2. a Current density-voltage-luminance traces. b Power efficiency-luminance-EQE traces

blue phosphorescence and TADF devices is currently reported.

\section{Materials and methods \\ Materials}

$\mathrm{MoO}_{3}, \mathrm{BCPO}, \mathrm{CzSi}$, TSPO1, TPBi, Bphen and LiF were purchased from Luminescence Technology Corp. TPEA was provided by the authors of literature ${ }^{35}$.

\section{Synthesis of Ce-1}

$\mathrm{KTp}^{\mathrm{Me} 2}(2.02 \mathrm{~g}, 6 \mathrm{mmol}), \mathrm{Ce}\left(\mathrm{CF}_{3} \mathrm{SO}_{3}\right)_{3}(1.17 \mathrm{~g}, 2 \mathrm{mmol})$, $\mathrm{H}_{2} \mathrm{O}(0.036 \mathrm{~g}, 2 \mathrm{mmol})$, and dry THF $(50 \mathrm{~mL})$ were added to a $100 \mathrm{~mL}$ round-bottom flask. The mixture was stirred in a glovebox at room temperature for 3 days. After filtering off the insoluble part, the solvent was removed under vacuum, and the resulting solid was loaded into a thermal sublimator. With a gradient temperature of $230-150-80^{\circ} \mathrm{C}$ and a pressure of $\sim 2 \times 10^{-4} \mathrm{~Pa}, 0.27 \mathrm{~g} \mathrm{Ce}-$ 1 was obtained as crystalline powder in $14 \mathrm{~h}$, with a yield of 15.8\%. Anal. calcd. for Ce-1: N 22.87\%; C 49.03\%; H 6.11\%; found: N 22.87\%; C 48.83\%; H 6.06\%. MALDI-HRICRMS calcd. for Ce-1 $\left[\mathrm{C}_{35} \mathrm{H}_{52} \mathrm{~B}_{3} \mathrm{CeN}_{14} \mathrm{O}\right]$ 857.3782, found $(\mathrm{M}+\mathrm{H})^{+}$858.3835. Crystallographic data of $\mathbf{C e - 1}$ (CCDC 1913494).

\section{General characterization}

Elemental analyses were performed on a VARIO EL analyser (GmbH, Hanau, Germany). High-resolution mass spectra were collected on a Bruker Solarix XR FTMS by the matrix-assisted laser desorption ionization (MALDI) method. UV-vis absorption spectra were recorded on a Shimadzu UV-3100 spectrometer. Fluorescence and transient PL decay spectra were measured on an Edinburgh Analytical Instruments FLS980 spectrophotometer. PLQYs were measured on a C9920-02 absolute quantum yield measurement system from Hamamatsu Company. Thermogravimetric analysis was undertaken with a Q600SDT instrument. Ultraviolet photoelectron spectroscopy was performed on an AXIS Supra X-ray photoelectron spectrometer.

\section{Density functional theory (DFT) calculations}

All calculations were performed with the ORCA programme package ${ }^{40}$. For ground state geometry optimizations, the hybrid B3LYP ${ }^{41-44}$ density functional was used without symmetry constraints. The all-electron triple- $\xi$ quality Def2-TZVP basis ${ }^{45}$ sets were assigned for B and N atoms. The Def2-ECP pseudopotential ${ }^{46}$ with Def2-TZVP valence basis sets was used for Ce (28 core electrons). Def2$\mathrm{SV}(\mathrm{P})$ basis sets ${ }^{47}$ were applied for the remaining elements in these compounds. The RI plus chain of spheres (RIJCOSX for B3LYP) approximation ${ }^{48}$ was used to accelerate the calculations with Weigend's "universal" Coulomb fitting auxiliary basis set def2 $/ \mathrm{J}^{49}$. We included the atom-pairwise dispersion correction with Becke-Johnson damping (D3BJ) to account for the van der Waals interaction ${ }^{50-53}$. In the single-point time-dependent DFT (TD-DFT) calculation, the B3LYP functional was applied with Def2-TZVP basis sets for all elements (the Def2-ECP pseudopotential was also applied for Ce).

\section{OLED fabrication and measurement}

Commercially available ITO-patterned anodes with a sheet resistance of $14 \Omega$ square $^{-1}$ and an 80 -nm thickness were used. ITO substrates were cleaned with deionized water and ethanol. The organic and metal layers were deposited in different vacuum chambers with a base pressure better than $1 \times 10^{-4} \mathrm{~Pa}$. The active area for each device was $4 \mathrm{~mm}^{2}$. All electric testing and optical measurements were performed under ambient conditions with encapsulation of devices in a glovebox. The EL spectra, current density-voltage-luminance $(J-V-L)$ characteristics, EQE characteristics and device lifetimes were measured by a computer-controlled Keithley 2400 source meter and an absolute EQE measurement 
system (C9920-12) with a photonic multichannel analyser (PMA-12, Hamamatsu Photonics).

\section{VASE method}

Doped films with a $20 \mathrm{~nm}$ thickness were deposited onto a clean quartz substrate for the VASE (ESM-300, J. A. Woollam Co.) measurement. The doped films were fabricated via thermal evaporation in a vacuum better than $1 \times 10^{-4} \mathrm{~Pa}$. The total deposition rate was $\sim 1 \AA \mathrm{s}^{-1}$, and the doping concentration of Ce-1 was $10 \mathrm{wt} \%$, consistent with the emission layer in OLEDs.

\section{Transient EL measurement}

Short-pulse excitation with a pulse width of $15 \mu$ s was generated using an Agilent 8114A. The amplitude of the pulse was $9 \mathrm{~V}$, and the baseline was $-3 \mathrm{~V}$. The period was $50 \mu \mathrm{s}$, the delay time was $25 \mu \mathrm{s}$, and the duty cycle was $30 \%$. The decay curves of the devices were detected using an Edinburgh FL920P transient spectrometer.

\section{EPR measurements}

cw-EPR spectra were measured on a Bruker Elexsys E580 spectrometer with a superhigh sensitivity probehead $(f=9.3757 \mathrm{GHz})$. The low-temperature environment was achieved by an Oxford Instruments ESR900 liquid helium cryostat.

\section{Acknowledgements \\ The authors gratefully acknowledge the financial support from the National Key R\&D Programme of China (Nos. 2017YFA0205100, 2016YFB0401001) and the Beijing Natural Science Foundation (2202015). Zifeng Zhao gratefully acknowledges financial support from the China Postdoctoral Science Foundation (2018M641065). The authors thank Prof. Lixin Xiao and Dr. Mengying Bian for providing TPEA and Mr. Yexin Wang for helping with the EPR test. This work is supported by the high-performance computing platform of Peking University.}

\section{Author details \\ ${ }^{1}$ Beijing National Laboratory for Molecular Sciences (BNLMS), State Key Laboratory of Rare Earth Materials Chemistry and Applications, Beijing Engineering Technology Research Centre of Active Display, College of Chemistry and Molecular Engineering, Peking University, 100871 Beijing, China. ${ }^{2}$ Tianjin Key Lab for Rare Earth Materials and Applications, School of Materials Science and Engineering, Nankai University, 300350 Tianjin, China. ${ }^{3}$ Department of Physics, Yunnan University, 2 Cuihu Bei Lu, 650091 Kunming China. ${ }^{4}$ Key Lab of Organic Optoelectronics and Molecular Engineering of Ministry of Education, Department of Chemistry, Tsinghua University, 100084 Beijing, China}

\section{Author contributions}

Z.Liu synthesized and characterized the compounds; L.W., Z.Z., and G.Z. collected and analysed the spectroscopic data; H.F. performed the theoretical calculation; H.Y. and N.J. performed the variable-angle spectroscopic ellipsometry; T.H., Y.Z., and L.D. measured the transient EL spectrum; L.W. and Z.Z. fabricated and tested the OLEDs; Z.Z. and Z.Liu wrote the manuscript. All authors discussed the results and commented on the manuscript. Z.Lu, Z.B., and C.H. directed the project.

\section{Conflict of interest}

L.W., Z.Z., Z.Liu, Z.B., and C.H. are inventors on a patent application based partly on the intellectual property in this report.
Supplementary information is available for this paper at https://doi.org/ 10.1038/s41377-020-00395-4.

Received: 9 April 2020 Revised: 24 August 2020 Accepted: 25 August 2020 Published online: 08 September 2020

\section{References}

1. Tang, C. W. \& Vanslyke, S. A. Organic electroluminescent diodes. Appl. Phys. Lett. 51, 913-915 (1987).

2. Ma, Y. G. et al. Electroluminescence from triplet metal-ligand charge-transfer excited state of transition metal complexes. Synth. Met. 94, 245-248 (1998).

3. Baldo, M. A. et al. Highly efficient phosphorescent emission from organic electroluminescent devices. Nature 395, 151-154 (1998).

4. Baldo, M. A. et al. Very high-efficiency green organic light-emitting devices based on electrophosphorescence. Appl. Phys. Lett. 75, 4-6 (1999).

5. Helander, M. G. et al. Chlorinated indium tin oxide electrodes with high work function for organic device compatibility. Science 332, 944-947 (2011).

6. Endo, A. et al. Thermally activated delayed fluorescence from Sn4+-porphyrin complexes and their application to organic light emitting diodes-a novel mechanism for electroluminescence. Adv. Mater. 21, 4802-4806 (2009).

7. Uoyama, H. et al. Highly efficient organic light-emitting diodes from delayed fluorescence. Nature 492, 234-238 (2012).

8. Hamze, R. et al. Eliminating nonradiative decay in $\mathrm{Cu}(\mathrm{l})$ emitters: $>99 \%$ quantum efficiency and microsecond lifetime. Science 363, 601-606 (2019).

9. Peng, Q. M. et al. Organic light-emitting diodes using a neutral $\pi$ radical as emitter: the emission from a doublet. Angew. Chem. 127, 7197-7201 (2015).

10. Obolda, A. et al. Up to $100 \%$ formation ratio of doublet exciton in deep-red organic light-emitting diodes based on neutral $\pi$-radical. ACS Appl. Mater. Interfaces 8, 35472-35478 (2016).

11. Ai, X. et al. Efficient radical-based light-emitting diodes with doublet emission. Nature 563, 536-540 (2018).

12. Yin, H. L. et al. Luminescent Ce(III) complexes as stoichiometric and catalytic photoreductants for halogen atom abstraction reactions. J. Am. Chem. Soc. 137, 9234-9237 (2015).

13. Yin, H. L. et al. Cerium photosensitizers: structure-function relationships and applications in photocatalytic aryl coupling reactions. J. Am. Chem. Soc. 138, 5984-5993 (2016).

14. Qiao, Y. S. et al. Understanding and controlling the emission brightness and color of molecular cerium luminophores. J. Am. Chem. Soc. 140, 4588-4595 (2018).

15. Lindqvist-Reis, P. et al. Unraveling the ground state and excited state structures and dynamics of hydrated $\mathrm{Ce}^{+}$ions by experiment and theory. Inorg. Chem. 57, 10111-10121 (2018).

16. Qin, $X$. et al. Lanthanide-activated phosphors based on $4 f-5 d$ optical transitions: theoretical and experimental aspects. Chem. Rev. 117, 4488-4527 (2017).

17. Wenger, O. S. Photoactive complexes with earth-abundant metals. J. Am. Chem. Soc. 140, 13522-13533 (2018).

18. Frey, S. T. \& Horrocks, W. D. Complexation, luminescence, and energy transfer of cerium(3+) with a series of multidentate aminophosphonic acids in aqueous solution. Inorg. Chem. 30, 1073-1079 (1991).

19. $\mathrm{Yu}, \mathrm{T}$. Z. et al. Ultraviolet electroluminescence from organic light-emitting diode with cerium(III)-crown ether complex. Solid-State Electron. 51, 894-899 (2007).

20. Zheng, X. L. et al. Bright blue-emitting Ce3+ complexes with encapsulating polybenzimidazole tripodal ligands as potential electroluminescent devices. Angew. Chem. Int. Ed. 46, 7399-7403 (2007).

21. Katkova, M. A. et al. Lanthanide imidodiphosphinate complexes: synthesis, structure and new aspects of electroluminescent properties. Synth. Met. 159, 1398-1402 (2009).

22. Trofimenko, S. Boron-pyrazole chemistry. J. Am. Chem. Soc. 88, 1842-1844 (1966).

23. Bünzli, J. C. G. \& Piguet, C. Taking advantage of luminescent lanthanide ions Chem. Soc. Rev. 34, 1048-1077 (2005).

24. Kunkely, H. \& Vogler, A. Can halides serve as a charge transfer acceptor? Metalcentered and metal-to-ligand charge transfer excitation of cerium(III) halides. Inorg. Chem. Commun. 9, 1-3 (2006).

25. Lee, J. et al. Deep blue phosphorescent organic light-emitting diodes with very high brightness and efficiency. Nat. Mater. 15, 92-98 (2016). 
26. Chou, H. H. \& Cheng, C. H. A highly efficient universal bipolar host for blue, green, and red phosphorescent OLEDs. Adv. Mater. 22, 2468-2471 (2010).

27. Yang, $\mathrm{H}$. et al. A phosphanthrene oxide host with close sphere packing for ultralow-voltage-driven efficient blue thermally activated delayed fluorescence diodes. Adv. Mater. 29, 1700553 (2017).

28. Li, X. Y. et al. Deep blue phosphorescent organic light-emitting diodes with ciey value of 0.11 and external quantum efficiency up to $22.5 \%$. Adv. Mater. 30, 1705005 (2018).

29. Schmidt, T. D. et al. Emitter orientation as a key parameter in organic lightemitting diodes. Phys. Rev. Appl. 8, 037001 (2017).

30. Kim, K. H. \& Kim, J. J. Origin and control of orientation of phosphorescent and TADF dyes for high-efficiency OLEDs. Adv. Mater. 30, 1705600 (2018).

31. Fleetham, T. et al. Efficient "pure" blue OLEDs employing tetradentate Pt complexes with a narrow spectral bandwidth. Adv. Mater. 26, 7116-7121 (2014).

32. Pal, A. K. et al. High-efficiency deep-blue-emitting organic light-emitting diodes based on iridium(III) carbene complexes. Adv. Mater. 30, 1804231 (2018).

33. Ahn, D. H. et al. Highly efficient blue thermally activated delayed fluorescence emitters based on symmetrical and rigid oxygen-bridged boron acceptors. Nat. Photonics 13, 540-546 (2019).

34. Liu, R. et al. Transient electroluminescence spikes in small molecular organic light-emitting diodes. Phys. Rev. B 83, 245302 (2011).

35. Bian, M. Y. et al. A combinational molecular design to achieve highly efficient deep-blue electrofluorescence. J. Mater. Chem. C 6, 745-753 (2018).

36. Schmidbauer, S., Hohenleutner, A. \& König, B. Chemical degradation in organic light-emitting devices: mechanisms and implications for the design of new materials. Adv. Mater. 25, 2114-2129 (2013).

37. Lin, N. et al. Molecular understanding of the chemical stability of organic materials for OLEDs: a comparative study on sulfonyl, phosphine-oxide, and carbonyl-containing host materials. J. Phys. Chem. C 118, 7569-7578 (2014).

38. Lin, N. et al. Achilles heels of phosphine oxide materials for OLEDs: chemical stability and degradation mechanism of a bipolar phosphine oxide/carbazole hybrid host material. J. Phys. Chem. C 116, 19451-19457 (2012).

39. Kusamoto, T. \& Nishihara, H. Efficiency breakthrough for radical LEDs. Nature 563, 480-481 (2018).

40. Neese, F. The ORCA program system. Wires Comput. Mol. Sci. 2, $73-78$ (2012).
41. Becke, A. D. Density-functional thermochemistry. III. The role of exact exchange. J. Chem. Phys. 98, 5648-5652 (1993).

42. Lee, C., Yang, W. T. \& Parr, R. G. Development of the colle-salvetti correlationenergy formula into a functional of the electron density. Phys. Rev. B 37, 785-789 (1988).

43. Vosko, S. H., Wilk, L. \& Nusair, M. Accurate spin-dependent electron liquid correlation energies for local spin density calculations: a critical analysis. Can. J. Phys. 58, 1200-1211 (1980).

44. Stephens, P. J. et al. Ab initio calculation of vibrational absorption and circular dichroism spectra using density functional force fields. J. Phys. Chem. 98, 11623-11627 (1994).

45. Weigend, F. \& Ahlrichs, R. Balanced basis sets of split valence, triple zeta valence and quadruple zeta valence quality for $\mathrm{H}$ to $\mathrm{Rn}$ : design and assessment of accuracy. Phys. Chem. Chem. Phys. 7, 3297-3305 (2005).

46. Andrae, D. et al. Energy-adjustedab initio pseudopotentials for the second and third row transition elements. Theor. Chim. Acta 77, 123-141 (1990).

47. Schäfer, A., Horn, H. \& Ahlrichs, R. Fully optimized contracted Gaussian basis sets for atoms Li to Kr. J. Chem. Phys. 97, 2571-2577 (1992).

48. Neese, F. et al. Efficient, approximate and parallel Hartree-Fock and hybrid DFT calculations. A 'chain-of-spheres' algorithm for the Hartree-Fock exchange. Chem. Phys. 356, 98-109 (2009).

49. Weigend, F. Accurate Coulomb-fitting basis sets for $\mathrm{H}$ to Rn. Phys. Chem. Chem. Phys. 8, 1057-1065 (2006).

50. Grimme, S., Ehrlich, S. \& Goerigk, L. Effect of the damping function in dispersion corrected density functional theory. J. Comput. Chem. 32, 1456-1465 (2011).

51. Grimme, S. Accurate description of van der Waals complexes by density functional theory including empirical corrections. J. Comput. Chem. 25, 1463-1473 (2004).

52. Grimme, S. Semiempirical GGA-type density functional constructed with a long-range dispersion correction. J. Comput. Chem. 27, 1787-1799 (2006).

53. Grimme, S. et al. A consistent and accurate $a b$ initio parametrization of density functional dispersion correction (DFT-D) for the 94 elements H-Pu. J. Chem. Phys. 132, 154104 (2010). 